\title{
Influence of Heat Treatment on the Conductivity of Nickel-Based Superalloys
}

\author{
Balasubramanian Nagarajan $^{1, a}$, Sylvie Castagne ${ }^{2, b}$,Swaminathan Annamalai ${ }^{1, \mathrm{c}}$,Zheng Fan ${ }^{2, d,}$ Chow Cher Wong ${ }^{3, \mathrm{e}}$ \\ ${ }^{1}$ Rolls-Royce@NTU Corporate Laboratory, c/o School of Mechanical \& Aerospace Engineering, 50 Nanyang Avenue, Nanyang \\ Technological University, Singapore 639798 \\ ${ }^{2}$ School of Mechanical and Aerospace Engineering, Nanyang Technological University, 50 Nanyang Avenue, Singapore 639798 \\ ${ }^{3}$ Rolls-Royce Singapore, Advanced Technology Centre, 6 Seletar Aerospace Crescent, Singapore 797575
}

\begin{abstract}
Non-destructive measurement of residual stress at the subsurface of nickel-based alloys using eddy current method has been limited by its sensitivity to its microstructure, especially to the precipitates. This paper investigates the effect of heat treatment on the electrical conductivity of RR1000, a nickel-based superalloy with a large fraction of $\gamma^{\prime}$ precipitates. Different heat treatment conditions, ranging from solution heat treatment to precipitation hardening with different aging times and temperatures, are used to achieve varying initial microstructures. Hardness of the samples is measured first to quantify the heat treated samples followed by the measurement of electrical conductivity using the conductivity probes of frequencies between $1 \mathrm{MHz}$ and $5 \mathrm{MHz}$. The relationship between the hardness and conductivity of the heat treated samples is then correlated further. The results highlight the significant influence of heat treatment on the sample hardness and the electrical conductivity of RR1000.
\end{abstract}

\section{Introduction}

Nickel-based alloys possess excellent corrosion resistance and mechanical strength, thus establishing them as ideal candidates for high temperature aerospace applications [1]. RR1000 is a Ni-based alloy developed by Rolls-Royce plc. using a powder metallurgy technique, which is used predominantly for turbine discs [2].

Fatigue life enhancement of the aerospace components can be achieved through inducing compressive residual stresses on the component surface and subsurface. However, non-destructive measurement of residual stresses after treatment or in-service components has been a challenge. Eddy current spectroscopy [3] is a suitable non-destructive method to measure residual stresses in nickel-based alloys presently. This method uses piezoresistivity effect, a relationship between the elastic stresses and the eddy current conductivity, to measure residual stresses in the material. Though this method is capable of detecting even minor changes in material conductivity induced by the residual stresses, it shows limitations through its sensitivity to microstructural features.

Abu-Nabah et al. [4] investigated the hardness influence on eddy current response of IN718 material through different heat treatment cycles. A significant correlation between bulk conductivity (measured as AECC, Apparent

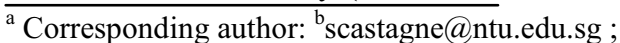

csannamalai@ntu.edu.sg;

dzfan@ntu.edu.sg;

echow.wong@Rolls-Royce.com
}

Eddy Current Conductivity) and hardness of the material is observed as both AECC and hardness increased monotonically with an increase in time and temperature of thermal aging. A similar behavior was observed by Chandrasekar et al. [5], where the linear relationship between hardness and conductivity of heat-treated IN718 prevailed. In a recent study, Pereira et al. [6] compared the hardness and the conductivity of IN718 with the lattice constant to examine the underlying mechanisms. It is consistently concluded from these research works [4-6] that the electrical conductivity of the material is influenced significantly by the evolution of precipitates during thermal aging. Formation and subsequent coarsening of $\gamma$ ', precipitates removes solute atoms from the matrix, causing a reduction in electron scattering and an increase in conductivity. However, overaging caused a reduction in conductivity resulting from the increased electron scattering due to the growth of plate-like $\delta$ phase precipitates [6]. It can be inferred from these results that the effect of microstructure and hardness effect on electrical conductivity in nickel-based superalloys is mainly dictated by $\gamma$ ' and $\delta$ phase precipitates, which are bct-ordered $\mathrm{Ni}_{3} \mathrm{Nb}$ and orthorhombic $\mathrm{Ni}_{3} \mathrm{Nb}$, respectively.

Fine grain RR1000 is comprised of a large volume of primary $\gamma$ ' precipitates but with no $\gamma$ ' and $\delta$ precipitates due to the absence of $\mathrm{Nb}$ in its composition. As the electrical 
conductivity is reported to be influenced significantly by $\gamma$ ', and $\delta$ precipitates, the absence of these precipitates in RR1000 could influence the electrical conductivity differently. Therefore, it is necessary to understand the influence of precipitation heat treatment on the conductivity of RR1000.

This paper studies the effect of thermal aging on electrical conductivity of RR1000 through conducting different heat treatments. Hardness values of various heat-treated samples are measured first. Electrical conductivity of samples is measured further using conductivity probes of different frequencies. Hardness and electrical conductivity are correlated for various heat treated conditions in order to understand the effect of thermal aging on the conductivity of RR1000.

\section{Materials and methods}

In this research, fine grain RR1000 provided by Rolls-Royce plc. was used. Table 1 lists the different heat treatment conditions used in this paper, based on previous heat treatments on RR1000 [7, 8]. The as-received sample had followed the process route of solution heat treatment, aging $\left(760^{\circ} \mathrm{C} / 16 \mathrm{hrs}\right)$ followed by forging. The as-received sample is represented as sample A. Samples B, C and D were solutionized first at $1115^{\circ} \mathrm{C}$ for $4 \mathrm{hrs}$ and then fan air quenched to room temperature. Then samples $\mathrm{C}$ and $\mathrm{D}$ were aged at $760^{\circ} \mathrm{C}$ and $620^{\circ} \mathrm{C}$ respectively for $8 \mathrm{hrs}$ before air cooling to room temperature. Two samples (with suffixes 1 and 2) were used in each of the heat treatment condition.

Table 1. Heat treatment conditions

\begin{tabular}{|c|c|}
\hline Sample & Heat treatment conditions \\
\hline $\mathrm{A}\left(A_{1}, A_{2}\right)$ & $\begin{array}{l}\text { As-received (solutionized, aged at } \\
\qquad 760^{\circ} \mathrm{C} / 16 \mathrm{hrs} \text { ) }\end{array}$ \\
\hline $\mathrm{B}\left(B_{1}, B_{2}\right)$ & $\begin{array}{c}\text { Solutionized at } 1115^{\circ} \mathrm{C} / 4 \mathrm{hrs} \text {, fan } \\
\text { air quenching }\end{array}$ \\
\hline $\mathrm{C}\left(C_{1}, C_{2}\right)$ & $\begin{array}{l}\text { Solutionized at } 1115^{\circ} \mathrm{C} / 4 \mathrm{hrs}, \\
\text { aged at } 760^{\circ} \mathrm{C} / 8 \mathrm{hrs} \text {, air cooling }\end{array}$ \\
\hline $\mathrm{D}\left(D_{1}, D_{2}\right)$ & $\begin{array}{l}\text { Solutionized at } 1115^{\circ} \mathrm{C} / 4 \mathrm{hrs}, \\
\text { aged at } 620^{\circ} \mathrm{C} / 8 \mathrm{hrs} \text {, air cooling }\end{array}$ \\
\hline
\end{tabular}

Electrical conductivity of the samples was measured using an eddy current system. The major components of the eddy current system are a portable Nortec 2000D+ eddy current test instrument, an absolute type Olympus surface probes covering a frequency bandwidth of 1-5 MHz, a multi-function DAQ to digitize analog signals, a PC based LabVIEW data acquisition software and two appropriate conductivity blocks for instrument calibration. The basic mode of operation of the eddy current equipment was in conductivity mode where the measured probe coil electrical impedance was transformed to another parameter known as apparent eddy current conductivity (AECC). Using two appropriate calibration blocks of known conductivities, four reference complex impedance points were measured with and without lift-off using a non-conducting shim of known thickness ( $\mathrm{t}$ ) between the probe coil and calibration block. Then the experimental measurements of complex coil impedance on the test specimen were evaluated in terms of AECC. Hardness of the samples was measured using a Vickers indenter with a load of $2000 \mathrm{Kgf}$. Hardness measurements were repeated 10 times and an average was calculated with error bars of one standard deviation shown in following analysis.

\section{Results and discussions}

\subsection{Hardness.}

The hardness of the samples for different heat treatments (as represented in Table 1) is measured to examine the effect of thermal aging on the hardening behavior. Fig. 1 compares the hardness values for different heat treatment conditions. It is evident from Fig. 1 that the minimum hardness (424.7 HV) was observed for sample B, which is in solution-annealed condition. As the solutionized state produces supersaturated solid solution and no precipitates, sample B has the lowest hardness as compared to that of other thermal aging conditions. It can be observed that the maximum hardness occurred for samples A (438.7 HV) and C $(438.8 \mathrm{HV})$. Due to the peak aging conditions of temperature and time at samples $\mathrm{A}$ and $\mathrm{C}$, the formation of precipitates and subsequent dispersion into the matrix is expected to occur. Due to the precipitation hardening, the hardness of the samples at these conditions (A and $\mathrm{C}$ ) is obviously higher. It is noted that the longer aging time with sample A has insignificant role on the resulting hardness. Sample D experienced underaging conditions $\left(620^{\circ} \mathrm{C}\right.$ temperature), where the hardness is lower than that of samples $\mathrm{A}$ and $\mathrm{C}$. The results indicate an apparent increase in hardness from the solution-annealed state to the precipitation hardened state. Despite the obvious change in hardness between the solutionized and aged samples, it can be noted from Fig. 1 that the hardness variation between different aging conditions has been insignificant.

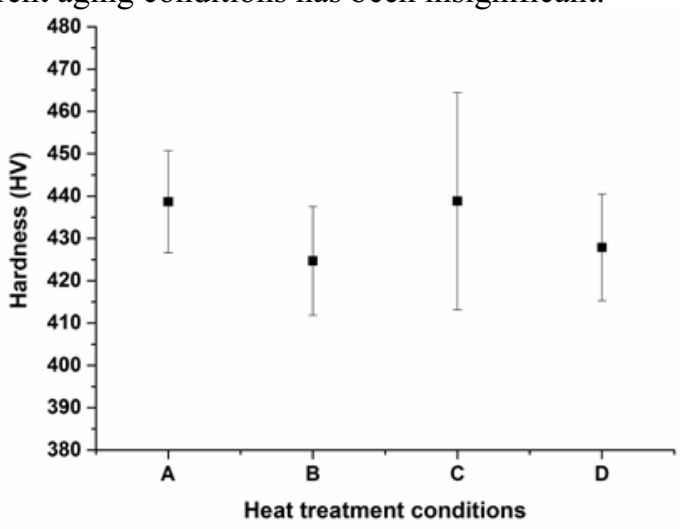

Figure 1. Effect of heat treatment on the hardness of RR1000 samples

\subsection{Electrical conductivity.}

Conductivity measurements were carried out on the RR1000 heat-treated samples using the eddy current system. The instrument is capable of determining the electrical conductivity of the samples as a percentage of the International Annealed Copper Standard (\%IACS) with an 
accuracy of $\pm 0.5 \%$ IACS or better. The electrical conductivities of the samples were measured for frequencies 1,3 and $5 \mathrm{MHz}$ and a minimum of 30 measurements were obtained at different times for each frequency. Fig 2 compares the conductivities of the two samples (with suffixes 1 and 2) at each heat treatment conditions for the three different frequencies. The error bars correspond to one standard deviation. The conductivities of the two samples, $\mathrm{A}_{1}$ and $\mathrm{A}_{2}$, for the similar heat treatment condition $\mathrm{A}$ has no variation and consistent across different frequencies. A similar behavior was also observed between the two samples for conditions B and C. It is found that the conductivity variation between different frequencies for all the samples except sample $D_{2}$ is much less than $1.0 \%$, which is within the maximum measurement error of the instrument $( \pm 0.5 \%)$. Despite the similar heat treatment condition, samples $\mathrm{D}_{1}$ and $\mathrm{D}_{2}$, show a large difference in conductivity across all three different frequencies. As the heat treated samples are expected to have uniform microstructure across their entire thickness, the conductivity should also remain constant with the change in frequency, which is inversely proportional to the penetration depth. This behavior could be attributed to the non-uniform formation and dispersion of precipitates during aging of RR1000 at the reduced temperature. However, measurements of the eddy-current conductivity within the frequency range tested, show a distinct difference in conductivity between the different heat treatment conditions, which is analyzed further.

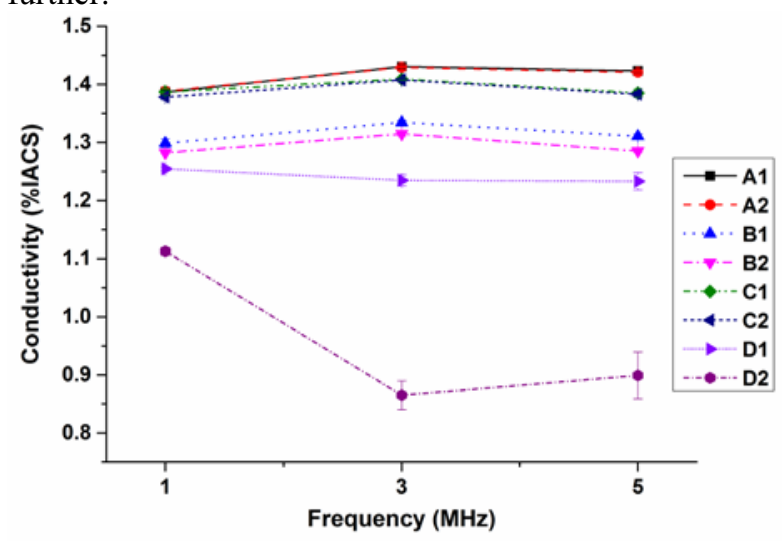

Figure 2. Electrical conductivity of heat-treated RR1000 samples as a function of frequency

Fig. 3 shows the average electrical conductivity of the heat-treated samples measured at the frequency of $1 \mathrm{MHz}$ along with the error bar of one standard deviation. An increase in conductivity was observed in the precipitation-hardened samples (A and C) as compared to the solution-annealed sample (B). The maximum conductivity was observed for samples $\mathrm{A}$ and $\mathrm{C}$, where peak aging temperatures were used. As the precipitates remove the solute atoms from the solid solution during aging, there will be less electron scattering in samples A and $\mathrm{C}$. Due to the scattering effect, the conductivity values are typically higher with the precipitation hardened samples. However, sample D has conductivity lower than that of the solutionized state (sample B) as seen in Figs. 2 and 3. A similar behavior of reduced conductivity was observed by
Chandrasekar et al. [5] with IN718 material due to the underaging heat treatment. This behavior was attributed to the possible formation of fine precipitates which resulted in the increased electron scattering and the reduced conductivity.

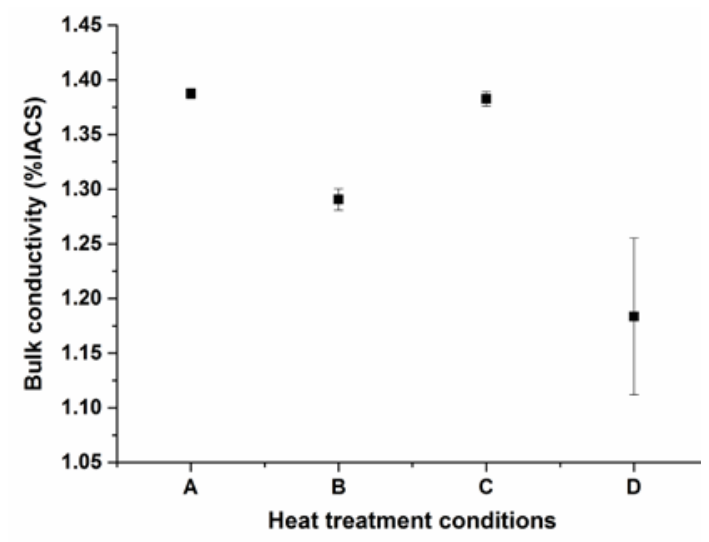

Figure 3. Effect of heat treatment on electrical conductivity at 1 $\mathrm{MHz}$ frequency

To evaluate the observed heat treatment effect on the conductivity, a comparison is made with the corresponding hardness values as shown in Fig. 4. The results clearly confirm that RR1000 exhibits the general monotonic trend between the hardness and the conductivity, as observed in the previous research works [4-6]. As mentioned earlier, the literature attributes the hardness influence of conductivity in nickel-based alloys on $\gamma^{\prime \prime}$ and $\delta$ phase precipitates. Despite the absence of these secondary precipitates, RR1000 showed significant variation in conductivity between different heat treatments.

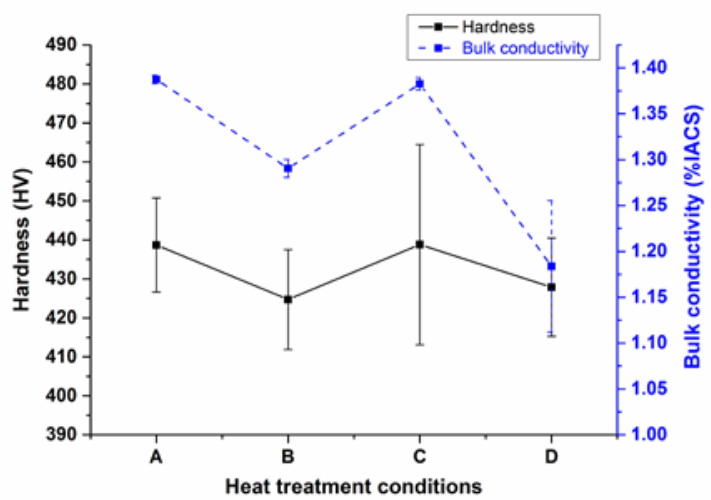

Figure 4. Correlation between conductivity and hardness of heat treated samples

\section{Conclusions}

This paper investigated the effect of thermal aging (precipitation treatment) on the electrical conductivity of RR1000. Different heat treatments ranging from solution-annealing to precipitation heat treatments were conducted on RR1000 samples, which were characterized by hardness and conductivity. The following conclusions can be drawn from the results: 
- An increase in hardness is observed with the precipitation-hardened samples compared to the solution-annealed sample. However, the time and temperature of thermal aging showed insignificant effect on the sample hardness.

- Precipitation hardening is found to increase the conductivity of RR1000 despite some discrepancies with the underaged sample.

- The hardness and conductivity of RR1000 demonstrated good correlation between the various heat treatment conditions.

Though the secondary phase precipitates are absent in RR1000, it exhibited significant conductivity deviation with the change in heat treatment conditions. Therefore, in order to examine the underlying mechanisms for the observed conductivity behavior with RR1000, further investigation of the corresponding variation in the material microstructure is required.

\section{Acknowledgements}

This work was conducted within the Rolls-Royce@NTU Corporate Lab with support from the National Research Foundation (NRF) Singapore under the Corp Lab@University Scheme.

\section{References}

1. Donachie, M.J. and S.J. Donachie, Superalloys - A Technical Guide (2nd Edition). ASM International.
2. Knowles, D.M. and D.W. Hunt, The influence of microstructure and environment on the crack growth behavior of powder metallurgy nickel superalloy RR1000. Metallurgical and Materials Transactions A, 2002. 33(10): p. 3165-3172.

3. Blodgett, M. and P. Nagy, Eddy Current Assessment of Near-Surface Residual Stress in Shot-Peened Nickel-Base Superalloys. Journal of Nondestructive Evaluation, 2004. 23(3): p. 107-123.

4. Abu-Nabah, B., et al., The Effect of Hardness on Eddy Current Residual Stress Profiling in Shot-Peened Nickel Alloys. Journal of Nondestructive Evaluation, 2010. 29(3): p. 143-153.

5. Chandrasekar, R., et al., Effects of Microstructure on Eddy Current Residual Stress Characterization of Shot-Peened Inconel 718. JOM, 2012. 64(2): p. 257-264.

6. Pereira, D., et al., Effect of microstructure on electrical conductivity of Inconel 718 alloys. Materials Science and Technology, 2015. 31(6): p. 669-676.

7. Pang, H., et al., Comparison of fatigue crack propagation in nickel base superalloys RR1000 and Udimet 720Li. Materials Science and Technology, 2015: p. 1743284715Y. 0000000060.

8. Birosca, S., et al., A quantitative approach to study the effect of local texture and heterogeneous plastic strain on the deformation micromechanism in RR1000 nickel-based superalloy. Acta Materialia, 2014. 74(0): p. 110-124. 